\title{
DFT STUDY OF GEOMETRIC ISOMERIZATION AND KETO- ENOL TAUTOMERIZATION OF DIHYDROXYFUMARIC ACID
}

\author{
NATALIA BOLOCAN ${ }^{\mathrm{a}, *}$, GHEORGHE DUCA ${ }^{\mathrm{b}}$
}

\begin{abstract}
A total of 45 isomers of dihydroxyfumaric acid (DHF), including 23 keto and 22 enediol forms, were identified and their geometrical isomerization and tautomerization was studied at the B3LYP level of theory using the $6-311++G(2 d f, 2 p)$ basis set in the gas phase and aqueous solution, and three most stable enediol structures were identified. Interconversions between the enediol forms and the keto forms proceed through two paths: (1) proton transfer $\left(E_{a} \approx 135-160 \mathrm{~kJ} \mathrm{~mol}^{-1}\right)$ and $(2)$ internal rotation $\left(E_{a} \approx 0.15-\right.$ $\left.75 \mathrm{~kJ} \mathrm{~mol}^{-1}\right)$. Keto-enol tautomeric reactions of dihydroxyfumaric acid were investigated for the three most stable enediol structures. It was found that the activation energy and the free activation energy is in the range of 230-310 $\mathrm{kJ} \mathrm{mol}^{-1}$ for the gas phase and by $50-80 \mathrm{~kJ} \mathrm{~mol}^{-1}$ lower in water, and TSs structures reveal that the carboxylic oxygen that forms the hydrogen bond in the enediol structure is involved in the mechanism of proton transfer. Furthermore, equilibrium constants have been calculated, along with the forward and reverse reaction rates for the isomerization and tautomerization reactions of the three most stable enediol structures, in gas and water.
\end{abstract}

Keywords: dihydroxifumaric acid, keto-enol tautomerism, dft study.

\section{INTRODUCTION}

Dihydroxyfumaric acid is the traditional name for (2E)-2,3dihydroxybut-2-enedioic acid, first obtained by Fenton in the 1890s [1]. It is an organic acid formed from tartaric acid by dehydrogenation or slow oxidation, a proven intermediate in the cycles of di- and tricarboxylic acids, and the glyoxalic acid via the tartaric acid transformation cycle.

\footnotetext{
a Laboratory of Physical and Quantum Chemistry, Institute of Chemistry, 3 Academiei str., Chisinau, MD 2028, Moldova

b Research Center of Physical and Inorganic Chemistry, Institute of Chemistry, 3 Academiei str., Chisinau, MD 2028, Moldova

*Corresponding author: natalia.bolocan@ichem.md
} 
An important intermediate in vegetal and living organisms, DHF recently became a molecule of interest in the scientific world, mainly due to Eschenmoser's proposal [2] that glyoxylate and DHF (its dimer) could have served as primary molecules in the synthesis of organic macromolecules in the constraints of prebiotic chemistry. Furthermore, our previous investigations showed that DHF and some of its salts and derivatives have wide practical applications and may be successfully used for the enhancement and preservation of wines [3,4], as inhibitors of nitrosoamines formation in vitro $[5,6]$ and in vivo $[7,8]$, as well as efficient scavengers of DPPH and ABTS free-radicals [9].

In spite of the interest recently attracted by DHF and its potential practical uses in various fields, to the best of our knowledge, to date there are no published theoretical investigations of the DHF conformational isomerism. Moreover, although there is evidence that keto-enol tautomerism is one of the most commonly studied forms of prototropy [10], studies of the DHF molecule are very sparse: keto-enol tautomerization of DHF acid was studied experimentally by Fleury and Souchay [11] in the 1960's, by Souza et al. in the 1980's during their investigations of DHF decarboxilation [12], and by Travin et al. in the 1980's during a kinetic investigation of the uranyl ion-DHF complex [13]. In this context, the present research was undertaken to provide valuable data for further use in both theoretical and practical areas.

In the case of DHF, various conformations are possible for the two tautomeric forms, due to the intramolecular rotations along the single $\mathrm{C}-\mathrm{C}$ bonds and due to the possible syn- and anti- periplanar orientation of the hydroxyl hydrogen with respect to the keto oxygen.

\section{RESULTS AND DISCUSSION}

\section{Thermodinamically stable isomers}

The calculated harmonic vibrational frequencies at the B3LYP/6$311++G(2 d f, 2 p)$ level of theory revealed that there are 22 enediol and 23 keto optimized structures of DHF at stationary points in the gas phase. The most stable enediol and keto structures are presented in Figure 1, and cartesian coordinates of all 45 optimized structures, for the gas phase and the aqueous medium, are available in Supplementary material. The isomers are nominated and arranged from the most stable isomer to least stable one (according to Gibbs free energy calculations). We nominate the enediol isomers as E1-E22, and the keto forms as K1-K23. 

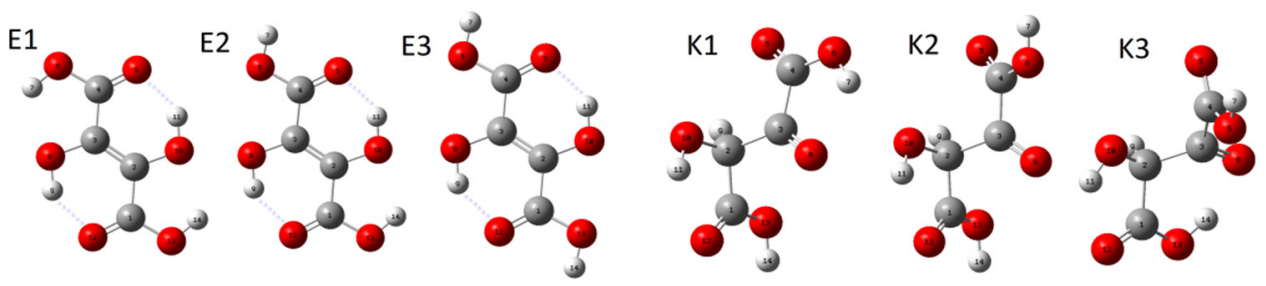

Figure 1. Optimized structures of the most stable enediol and keto isomers of dihydroxyfumaric acid

The electronic energy $(E)$, enthalpy $(H)$ and Gibbs energy $(G)$ of each conformer were calculated at $298.15 \mathrm{~K}$. Using the energy of E1 as the reference point for gas calculations, and the energy of E2 as the reference point for calculations in the aqueous medium, all values obtained for $\Delta E, \Delta H$ and $\Delta G$ are listed together in Table 1 for comparison.

Table 1. Relative energies $\left(\mathrm{kJ} \mathrm{mol}^{-1}\right)$ with respect to the most stable enediol (E1), of the enediol (E1-E22) and keto (K1-K23) isomers of dihydroxyfumaric acid, at $298.15 \mathrm{~K}$. In gas phase, the total energy values for E1 are as follows: $E=-606.1440772$ a.u., $H=-606.04156958$ a.u., $G=-606.08507874$ a.u. In water, the total energy values for $E 2$ are as follows: $E=-606.15927423$ a.u., $H=--606.05902544$ a.u., $G=-606.10366354$ a.u.

\begin{tabular}{|c|c|c|c|c|c|c|c|c|c|c|c|c|c|}
\hline \multirow[t]{2}{*}{$\begin{array}{l}\text { Enediol } \\
\text { isomers }\end{array}$} & \multicolumn{2}{|c|}{$\Delta E$} & \multicolumn{2}{|c|}{$\Delta H$} & \multicolumn{2}{|c|}{$\Delta G$} & \multirow[t]{2}{*}{$\begin{array}{c}\text { Keto } \\
\text { isomers }\end{array}$} & \multicolumn{2}{|c|}{$\Delta E$} & \multicolumn{2}{|c|}{$\Delta H$} & \multicolumn{2}{|c|}{$\Delta G$} \\
\hline & Gas & Water & Gas & Water & Gas & Water & & Gas & Water & Gas & Water & Gas & Water \\
\hline E1 & 0.00 & 0.59 & 0.00 & 0.53 & 0.00 & & $K 1$ & 54.34 & & & & 46.70 & 32.52 \\
\hline$E 2$ & 5.18 & 0.00 & 5.26 & 0.00 & 5.07 & 0.00 & $K 2$ & 58.64 & 35.61 & 56.85 & 38.70 & 49.52 & 33.92 \\
\hline E3 & 10.42 & 0.01 & 10.54 & 0.58 & 10.05 & 1.24 & K3 & 58.32 & 39.02 & 55.89 & 40.65 & 50.63 & 36.63 \\
\hline E4 & 15.46 & 10.54 & 15.89 & 10.62 & 14.90 & 7.59 & K4 & 63.37 & 37.36 & 61.15 & 40.60 & 53.27 & 35.28 \\
\hline E5 & 24.33 & 16.07 & 24.74 & 121.6 & 22.99 & 12.41 & K5 & 60.67 & 36.20 & 59.55 & 40.97 & 54.84 & 39.55 \\
\hline E6 & 26.66 & 9.24 & 26.21 & 9.40 & 25.05 & & K6 & 64.46 & 39. & 63.45 & 43.18 & & 39.9 \\
\hline E7 & 30.59 & 9.86 & 30.13 & 10.23 & 28.65 & 9.7 & $K 7$ & 62.83 & 39.97 & 61.77 & 42.57 & & 37.42 \\
\hline E8 & 39.95 & 21.39 & 36.73 & 22.46 & 37.36 & 20.86 & $K 8$ & 63.22 & 37.76 & 61.88 & 40.41 & 56.57 & 36.34 \\
\hline E9 & 38.07 & 21.40 & 38.45 & 22.44 & 35.88 & 20.64 & K9 & 69.15 & 40.00 & 67.44 & 43.32 & 59 & 39.07 \\
\hline E10 & 40.46 & 19.58 & 39.97 & 22.15 & 36.67 & 21. & K10 & 62.96 & 37. & 61.29 & 38 & & 38.91 \\
\hline E11 & 41.07 & 15.73 & 40.10 & 17.01 & 37.41 & 17. & K11 & 72.92 & 42.22 & 70.65 & 45.44 & 62.30 & 38.46 \\
\hline E12 & 42.66 & 16.56 & 41.89 & 17.55 & 38.49 & 17. & K12 & 68.81 & 36 & & & 62.73 & 37.5 \\
\hline E13 & 44.21 & 16.92 & 43.85 & 18.60 & 41.44 & 19.35 & K13 & 71.95 & 39.77 & 70.31 & 42.69 & 63.04 & 37.76 \\
\hline E14 & 44.48 & 18.59 & 41.31 & 20.11 & 42.81 & 20.1 & K14 & 70.45 & 35.84 & 68.97 & 35.38 & 63.36 & 35.64 \\
\hline E15 & 50.37 & 21.17 & 49.91 & 23.43 & 46.55 & 23. & K15 & 72.24 & 38.18 & 70.39 & 40.77 & 64.11 & 35.20 \\
\hline E16 & 51.27 & 22.88 & 50.94 & 24.73 & 47.16 & 24. & K16 & 78.83 & 46.30 & 76.76 & 48.72 & 69.98 & 50.32 \\
\hline E17 & 51.07 & 21.12 & 50.42 & 22.72 & 47.60 & 23. & & 82.73 & 41.31 & 80.28 & 44.87 & 71.19 & 39.40 \\
\hline E18 & 51.53 & 20.59 & 50.65 & 21.96 & 48.16 & 22. & K18 & 80.28 & 40 & 77.79 & 42.13 & 72.48 & 36.5 \\
\hline E19 & 52.67 & 26.93 & 52.13 & 29.29 & 48.46 & 29.32 & K19 & 83.40 & 39.57 & 80.76 & 41.98 & 73.69 & 36.00 \\
\hline E20 & 59.99 & 19.98 & 59.70 & 22.15 & 58.37 & 23.85 & $K 20$ & 80.71 & 45.73 & 78.31 & 47.93 & 74.03 & 46.49 \\
\hline E21 & 66.16 & 33.48 & 64.68 & 34.09 & 60.06 & 32.14 & $K 21$ & 83.82 & 37.52 & 81.62 & 40.73 & 74.26 & 36.99 \\
\hline \multirow[t]{2}{*}{$E 22$} & 72.94 & 27.95 & 72.89 & 32.85 & 70.64 & 33.78 & $K 22$ & 82.55 & 44.38 & 80.86 & 46.49 & 75.24 & 43.81 \\
\hline & & & & & & & $K 23$ & 112.03 & 56.13 & 110.6 & 59.01 & 104.8 & 57.63 \\
\hline
\end{tabular}


Results show that the enediol forms of DHF are more stable than the keto forms, both in gas phase and in water.

In the gas phase, the enediol structure $\mathrm{E} 1$ of DHF is the most stable form and represents the global minimum in the potential energy curve of DHF acid. It should be mentioned here that its Gibbs free energy is lower than that of the most stable keto structure $\mathrm{K} 1$ by $46.7 \mathrm{~kJ} \mathrm{~mol}^{-1}$ at the DFT(B3LYP) level. The stability of E1 may be attributed to the presence of the intramolecular hydrogen bonding effect present in the E1 structure more than in others, and to stabilizing orbital interactions due to the anti- periplanar orientation of the hydroxyl hydrogen with respect to the keto oxygen.

In water, the most stable isomer is E2. It should be mentioned here that its Gibbs free energy is lower than that of the most stable keto structure $\mathrm{K} 1$ by $32.7 \mathrm{~kJ} \mathrm{~mol}^{-1}$ at the DFT(B3LYP) level.

The Boltzmann distribution according to Eq. 1. shows that in gas phase, $99.78 \%$ of DHF are represented by only 3 enediol structures: the most stable isomer E1 accounts for $87.4 \%$, followed by isomer E2 with a relative abundance of $10.98 \%$, then isomer E3 $-1.4 \%$. In aqueous solution, the three most stable enediolic forms account for $97.3 \%$ of the acid, with the following relative abundance indices: the most stable isomer E2 accounts for $38.4 \%$, followed by E1 - $31.8 \%$ and E3 - $27.1 \%$.

\section{Comparison of some geometrical parameters of enediol and keto structures}

The enediol structures of DHF are almost completely planar, except for structures E20 and E22, which suffer from atom-atom repulsion. This planarity of enediol structures is probably a consequence of intramolecular interactions between the enolic $-\mathrm{OH}$ groups and the $\mathrm{C}=\mathrm{O}$ or $-\mathrm{OH}$ of the carboxylic groups which lead to electron delocalization in the molecule. Selected geometrical parameters for the four most stable enediol and keto structures are available in Supporting information.

The three most stable enediol structures of DHF are characterized by dihedral angles deviations of no more than $0.1^{\circ}$, while in all other enediol isomers structures (except for E20 and E22) the discussed dihedral angles deviations are a bit larger, but do not exceed $1^{\circ}$ from planarity. The most significant deviations are present in structures E20 and E22: up to $-3.8^{\circ}$ and $-2.4^{\circ}$ for the $(\mathrm{O} 8 \mathrm{C} 3 \mathrm{C} 2 \mathrm{C} 1)$ angle, $-16.1^{\circ}$ and $-3.8^{\circ}$ for the (O8C3C4O5) angle, $-17.3^{\circ}$ and $-3.2^{\circ}$ for the $\left(\mathrm{O} 6 \mathrm{C} 4 \mathrm{C} 3 \mathrm{C} 2\right.$ ) angle and $-179.1^{\circ}$ and $-166.8^{\circ}$ for the $(\mathrm{O} 10 \mathrm{C} 2 \mathrm{C} 1 \mathrm{O} 13)$ angle. E20 and E22 are the only enediol structures which deviate significantly from planarity because the anti- periplanar oriented hydroxyl hydrogens (with respect to the keto oxygen) of the two carboxylic 
groups on both ends of the molecule force the enolic hydrogen atom out of the plane almost perpendicularly, with the $(\mathrm{C} 1 \mathrm{C} 2 \mathrm{O} 10 \mathrm{H} 11)$ dihedral angle being $94.1^{\circ}$ and $72.9^{\circ}$ for the E20 and E22, respectively.

TABLE 2. Select dihedral angles (in ${ }^{\circ}$ ) and dipole moments (in Debye) of the four most stable enediol and four most stable keto isomers of DHF, in gas

\begin{tabular}{|cccccc|}
\hline Isomer & $\begin{array}{c}\text { dihedral } \\
\text { (O10C3C2O8) } \\
\text { angle }\end{array}$ & $\begin{array}{c}\text { dihedral } \\
\text { (O10C2C1O12) } \\
\text { angle }\end{array}$ & $\begin{array}{c}\text { dihedral } \\
\text { (O10C3C2C1) } \\
\text { angle }\end{array}$ & $\begin{array}{c}\text { dihedral } \\
\text { (O5C4C3O8) } \\
\text { angle }\end{array}$ & $\begin{array}{c}\text { Dipole } \\
\text { moment }\end{array}$ \\
E1 & 180.0 & -0.1 & -0.0 & 180.0 & 0.00132 \\
E2 & 180.0 & -0.0 & -0.1 & -180.0 & 3.10558 \\
E3 & 180.0 & -0.0 & -0.0 & 180.0 & 0.00107 \\
E4 & 179.9 & -180.0 & -0.1 & 0.1 & 0.00262 \\
K1 & 93.6 & -6.2 & -26.8 & -8.9 & 3.17906 \\
K2 & 116.1 & -35.8 & -4.5 & -13.6 & 0.81446 \\
K3 & 171.8 & -82.8 & 47.0 & 11.6 & 1.66599 \\
K4 & 130.6 & 133.8 & 9.0 & -18.3 & 3.19301 \\
\hline
\end{tabular}

The keto structures are twisted with the dihedral (010C2C3O8) angle ranging between $88.8^{\circ}$ to $175.6^{\circ}$. The other dihedral angles deviate from planarity by up to $85.7^{\circ}$ in some keto structures. This variation of the dihedral angles, however, only slightly influences other bond lengths and angles.

\section{Barriers of Rotation and Tautomerization}

Transition states of interconversions between the enediol and the keto isomers

All TS structures related to the interconversion of the enediol forms and the keto forms of DHF acid were identified, and select TSs are presented in Figure 2. Table 3 presents the activation energy $\left(E_{a}\right)$, imaginary frequency $(v)$, and Gibbs free activation energy $\left(\Delta G^{\#}\right)$ for interconversions of select enediol and keto forms. The enthalpy activation energy $\left(\Delta H^{\#}\right)$ has been calculated as well, and its values were almost equal to $\Delta G^{\#}$.

Interconversions between the enediol forms and between the keto forms take place through both proton transfer and internal rotation $(\mathrm{C}-\mathrm{C}$ and $\mathrm{O}-\mathrm{H}$ rotation) pathways. In enediol-enediol transformations, the activation energies of proton transfer are in the range of $135-160 \mathrm{~kJ} \mathrm{~mol}^{-1}$ and for the rotational path are in the range $5-75 \mathrm{~kJ} \mathrm{~mol}^{-1}$. For interconversions between the keto forms, activation energies are in the range of $139-153 \mathrm{~kJ} \mathrm{~mol}^{-1}$ for the proton transfer path and of $0.15-52 \mathrm{~kJ} \mathrm{~mol}^{-1}$ for the rotational path. 

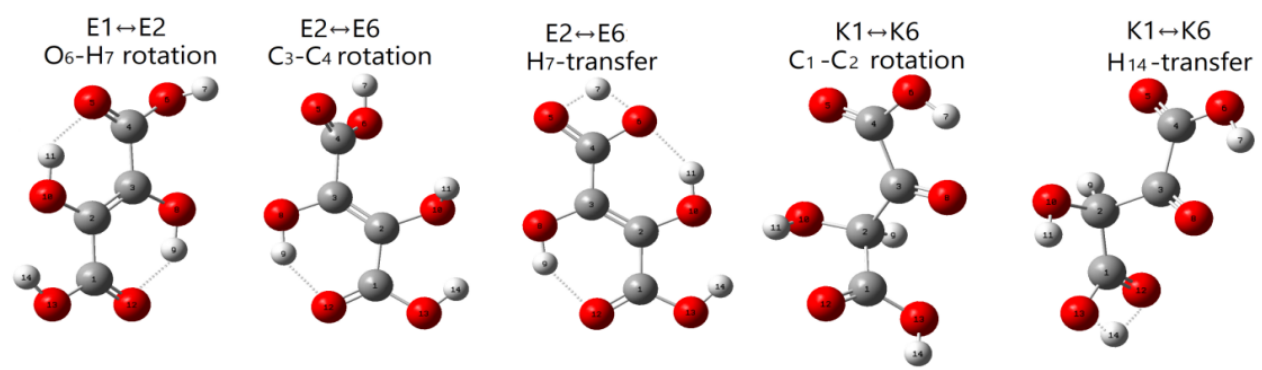

Figure 2. Optimized geometries of selected TSs of enediol-enediol and keto-keto interconversions

In the case of enediol and keto isomers bearing at least one carboxylic group with a syn- periplanar oriented hydrogen, there are a number of transformations that take place through both pathways, proton transfer and $\mathrm{C}-\mathrm{C}$ rotation, and these interconversions may be regarded as competitive reactions.

These are: E2 $\leftrightarrow \mathrm{E} 6, \mathrm{E} 3 \leftrightarrow \mathrm{E} 7, \mathrm{E} 5 \leftrightarrow \mathrm{E} 10, \mathrm{E} 7 \leftrightarrow \mathrm{E} 18, \mathrm{E} 8 \leftrightarrow \mathrm{E} 13, \mathrm{E} 9$ $\leftrightarrow \mathrm{E} 19, \mathrm{E} 11 \leftrightarrow \mathrm{E} 17, \mathrm{E} 12 \leftrightarrow \mathrm{E} 14, \mathrm{E} 12 \leftrightarrow \mathrm{E} 15, \mathrm{E} 14 \leftrightarrow \mathrm{E} 16, \mathrm{E} 15 \leftrightarrow \mathrm{E} 16, \mathrm{E} 19$ $\leftrightarrow \mathrm{E} 21$ and $\mathrm{K} 1 \leftrightarrow \mathrm{K} 6, \mathrm{~K} 2 \leftrightarrow \mathrm{K} 4, \mathrm{~K} 2 \leftrightarrow \mathrm{K} 9, \mathrm{~K} 4 \leftrightarrow \mathrm{K} 11, \mathrm{~K} 7 \leftrightarrow \mathrm{K} 8, \mathrm{~K} 9 \leftrightarrow \mathrm{K} 11$, $\mathrm{K} 12 \leftrightarrow \mathrm{K} 22, \mathrm{~K} 13 \leftrightarrow \mathrm{K} 15, \mathrm{~K} 13 \leftrightarrow \mathrm{K} 17, \mathrm{~K} 14 \leftrightarrow \mathrm{K} 21, \mathrm{~K} 15 \leftrightarrow \mathrm{K} 19, \mathrm{~K} 17 \leftrightarrow \mathrm{K} 19$. However, the activation energies for $\mathrm{H}$-transfer processes are significantly higher than for the $\mathrm{C}-\mathrm{C}$ rotational path, and the ratios of $\mathrm{k}_{\mathrm{p}} / \mathrm{k}_{\mathrm{r}}\left(\mathrm{k}_{\mathrm{p}}\right.$ and $\mathrm{k}_{\mathrm{r}}$ are the rate constants of the proton transfer and the rotational paths, respectively) are obtained zero at $298.15 \mathrm{~K}$ in the gas phase, based on the Arrhenius rate constant equation, if the same value of $A$ is considered for both paths.

Therefore, we may conclude that the above-mentioned interconversions take place in gas phase through the $\mathrm{C}-\mathrm{C}$ rotational paths, and proton transfer processes can not occur simultaneously.

Imaginary frequencies $\left(v\right.$, in $\left.\mathrm{cm}^{-1}\right)$ of select TS structures for enediolenediol and keto-keto interconversions are tabulated in Table 3. As expected, the $\mathrm{C}-\mathrm{C}$ internal rotation has the minimum frequency (around $-40 \mathrm{~cm}^{-1}$ $100 \mathrm{~cm}^{-1}$ ), being almost a pure rotational vibration. Also, the imaginary frequency of the proton transfer reaction is around $-1900 \mathrm{~cm}^{-1}$, a stretching vibration frequency. In the case of $\mathrm{O}-\mathrm{H}$ rotation, the imaginary frequency values are between pure rotation and pure stretching vibrations, usually in the range of $-200 \mathrm{~cm}^{-1} \ldots-600 \mathrm{~cm}^{-1}$, slightly higher in the case of carboxylic $\mathrm{O}-\mathrm{H}$ rotations. This is probably due to the fact that there are a lot of hydrogen bonds/interactions and electron delocalization sites in DFH isomers, and the rotation of a hydrogen atom may break a hydrogen bond and start the formation of another hydrogen bond, therefore, the $\mathrm{O}-\mathrm{H}$ rotation is mixed with a stretching vibration and its 
DFT STUDY OF GEOMETRIC ISOMERIZATION AND KETO-ENOL TAUTOMERIZATION OF ...

Table 3. Activation energy, $E_{a}$, Gibbs free activation energy, $\Delta G^{\#}\left(\mathrm{KJ} \mathrm{mol}^{-1}\right)$, and imaginary frequency, $v\left(\right.$ scaled by $\left.0.967^{16}\right)\left(\mathrm{cm}^{-1}\right)$, for select interconversions between enediol isomers and between the keto forms.

\begin{tabular}{|c|c|c|c|c|c|c|}
\hline \multicolumn{7}{|c|}{ Enediol-enediol interconversions } \\
\hline \multirow[t]{2}{*}{ TS } & \multicolumn{2}{|c|}{$\mathrm{v}$} & \multicolumn{2}{|c|}{$E_{a}$} & \multicolumn{2}{|c|}{$\Delta G^{\#}$} \\
\hline & \multicolumn{6}{|c|}{$\mathrm{O}-\mathrm{H}$ rotation } \\
\hline $\begin{array}{l}\mathrm{E} 1 \rightarrow \mathrm{E} 2 \\
\mathrm{~F} 2 \rightarrow \mathrm{F} 1\end{array}$ & \multirow{2}{*}{-607.01} & \multirow[t]{2}{*}{-426.97} & 52.19 & 41.42 & 47.50 & 39.90 \\
\hline $\begin{array}{l}\mathrm{E} 2 \rightarrow \mathrm{E} 1 \\
\mathrm{~F} 2 \rightarrow \mathrm{F} 3\end{array}$ & & & 47.01 & 42.01 & 42.43 & 40.38 \\
\hline $\mathrm{E} 2 \rightarrow \mathrm{E} 3$ & \multirow{2}{*}{-601.56} & \multirow{2}{*}{-575.23} & 51.71 & 41.52 & 47.01 & 40.19 \\
\hline $\mathrm{E} 3 \rightarrow \mathrm{E} 2$ & & & 46.47 & 41.53 & 42.03 & 38.96 \\
\hline $\mathrm{E} 6 \rightarrow \mathrm{E} 17$ & \multirow{3}{*}{-374.78} & \multirow{2}{*}{-324.1} & 45.17 & 30.02 & 40.43 & 29.94 \\
\hline $\mathrm{E} 17 \rightarrow \mathrm{E} 6$ & & & 20.76 & 18.13 & 17.87 & 15.66 \\
\hline \multicolumn{6}{|c|}{$\mathrm{C}-\mathrm{C}$ rotation } & \\
\hline $\begin{array}{l}\mathrm{E} 9 \rightarrow \mathrm{E} 19 \\
\mathrm{E} 19 \rightarrow \mathrm{E} 9\end{array}$ & -46.23 & -62.20 & $\begin{array}{l}48.11 \\
37.24\end{array}$ & $\begin{array}{l}34.25 \\
26.47\end{array}$ & $\begin{array}{c}65.83 \\
7.46\end{array}$ & $\begin{array}{l}33.71 \\
25.32\end{array}$ \\
\hline $\mathrm{E} 2 \rightarrow \mathrm{E} 6$ & \multirow{3}{*}{-52.04} & \multirow{2}{*}{-72.12} & 52.64 & 33.45 & 49.31 & 35.09 \\
\hline $\mathrm{E} 6 \rightarrow \mathrm{E} 2$ & & & 31.15 & 24.21 & 29.34 & 26.36 \\
\hline \multicolumn{6}{|c|}{$\mathrm{H}$-transfer } & \\
\hline $\mathrm{E} 2 \rightarrow \mathrm{E} 6$ & \multirow{2}{*}{$-1,875.32$} & \multirow{2}{*}{-1997.15} & 158.92 & 164.36 & 145.82 & 154.28 \\
\hline $\mathrm{E} 6 \rightarrow \mathrm{E} 2$ & & & 137.43 & 155.12 & 125.85 & 145.54 \\
\hline $\mathrm{E} 3 \rightarrow \mathrm{E} 7$ & \multirow{2}{*}{$-1,873.71$} & \multirow{2}{*}{-1995.14} & 156.93 & 163.68 & 143.84 & 152.10 \\
\hline $\mathrm{E} 7 \rightarrow \mathrm{E} 3$ & & & 136.76 & 153.81 & 125.23 & 143.56 \\
\hline \multicolumn{7}{|c|}{ Keto-keto interconversions } \\
\hline TS & \multicolumn{2}{|c|}{$\mathrm{v}$} & \multicolumn{2}{|c|}{$E_{a}$} & \multicolumn{2}{|c|}{$\Delta G^{\#}$} \\
\hline & Gas & Water & Gas & Water & Gas & Water \\
\hline \multicolumn{7}{|c|}{$\mathrm{O}-\mathrm{H}$ rotation } \\
\hline $\begin{array}{l}\mathrm{K} 1 \rightarrow \mathrm{K} 2 \\
\mathrm{~K} 2 \rightarrow \mathrm{K} 1\end{array}$ & -487.93 & -520.06 & $\begin{array}{l}48.76 \\
44.46\end{array}$ & $\begin{array}{l}44.06 \\
43.11\end{array}$ & $\begin{array}{l}43.87 \\
41.04\end{array}$ & $\begin{array}{l}39.81 \\
38.41\end{array}$ \\
\hline $\mathrm{K} 1 \rightarrow \mathrm{K} 16$ & & & 44.33 & 43.50 & 40.64 & 37.71 \\
\hline $\mathrm{K} 16 \rightarrow \mathrm{K} 1$ & -492.33 & -507.37 & 28.18 & 31.86 & 25.91 & 19.91 \\
\hline $\mathrm{K} 11 \rightarrow \mathrm{K} 22$ & & & 41.57 & 41.00 & 37.88 & 36.35 \\
\hline $\mathrm{K} 22 \rightarrow \mathrm{K} 11$ & -481.19 & -536.29 & 31.94 & 38.83 & 24.94 & 31.00 \\
\hline & & & rotation & & & \\
\hline $\mathrm{K} 1 \rightarrow \mathrm{K} 6$ & & -56.36 & 22.84 & 14.15 & 23.87 & 15.10 \\
\hline $\mathrm{K} 6 \rightarrow \mathrm{K} 1$ & -73.29 & -56.30 & 12.72 & 9.32 & 14.19 & 7.71 \\
\hline $\mathrm{K} 1 \rightarrow \mathrm{K} 12$ & & & 23.62 & 17.50 & 23.73 & 18.20 \\
\hline $\mathrm{K} 12 \rightarrow \mathrm{K} 1$ & -69.77 & -215.90 & 9.15 & 15.59 & 7.70 & 13.13 \\
\hline & & & transfer & & & \\
\hline $\mathrm{K} 1 \rightarrow \mathrm{K} 6$ & & -193646 & 162.29 & 167.03 & 148.58 & 156.83 \\
\hline $\mathrm{K} 6 \rightarrow \mathrm{K} 1$ & 1,881.85 & -1936.46 & 152.17 & 162.20 & 138.90 & 149.44 \\
\hline $\mathrm{K} 2 \rightarrow \mathrm{K} 4$ & & & 167.09 & 168.40 & 153.19 & 155.58 \\
\hline $\mathrm{K} 4 \rightarrow \mathrm{K} 2$ & $-1,881$. & -1938.56 & 162.36 & 166.65 & 149.43 & 154.22 \\
\hline
\end{tabular}


frequency is higher than that for $\mathrm{C}-\mathrm{C}$ rotational vibration. Calculation results for the aqueous medium show that imaginary frequencies for $\mathrm{O}-\mathrm{H}$ and $\mathrm{C}-\mathrm{C}$ rotations are lower in water than in gas, while for $\mathrm{H}$-transfer reactions, imaginary frequencies are higher in water than in gas, both for enediolenediol and keto-keto interconversions. Also, the activation energies of $\mathrm{H}$ transfer reactions are higher for water, than for gas, probably due to the stabilization of the structures by additional hydrogen bonds with water molecules from the implicit solvent medium.

\section{Keto-enol tautomerism of DHF acid}

Keto-enol tautomerization was investigated for the three most stable enediol structures that account for $99.76 \%$ of DHF in the gas phase, and $97.3 \%$ in aqueous medium. Optimized TS structures in gas phase are shown in Fig. 3, and all cartesian coordinates are presented in supplementary material.
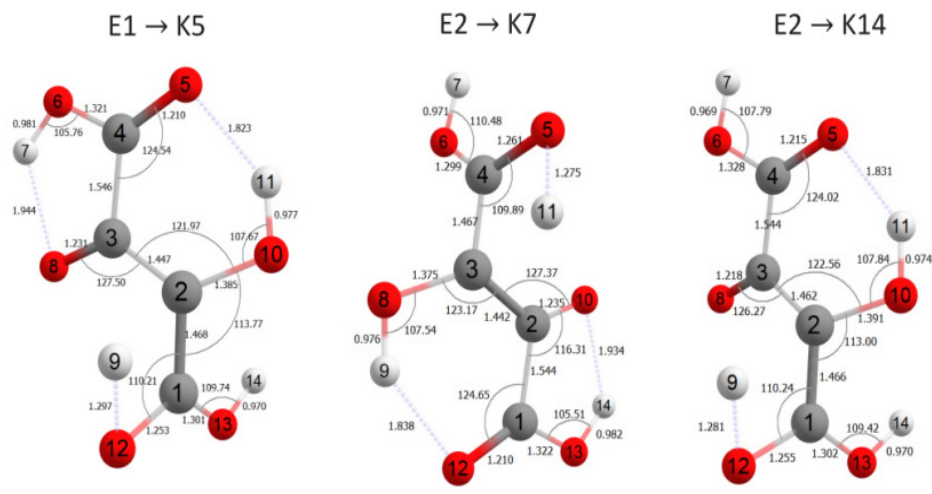

$$
\mathrm{E} 3 \rightarrow \mathrm{K} 13
$$

Figure 3. Optimized TS structures of TSs in the keto-enol tautomerism of the three most stable enediol isomers of DHF, in the gas phase

Energy barriers of the keto-enol tautomerization process were computed using the energy differences between local minimum structures (Table 1) and transition states. The only route for the keto-enol tautomerism is through proton transfer. Table 4 presents the activation energy, $E_{a}$, imaginary frequency, $v$, and Gibbs free activation energy, $\Delta G^{\#}$ of the keto-enol interconversions calculated at the B3LYP level of theory at $298.15 \mathrm{~K}$. As expected, the barriers for enediol $\rightarrow$ keto conversions exceed those for the reverse keto $\rightarrow$ enediol transitions, in gas and in water, by around $50 \mathrm{~kJ} \mathrm{~mol}^{-1}$. 
DFT STUDY OF GEOMETRIC ISOMERIZATION AND KETO-ENOL TAUTOMERIZATION OF ...

Table 4. Activation energy, $E_{a}$, imaginary frequency, $v$ (scaled by $\left.0.967^{16}\right)\left(\mathrm{cm}^{-1}\right)$, and Gibbs free activation energy, $\Delta G^{\#}$, for the keto-enol tautomerism of the three most stable enediol forms, in gas and solvent (water).

\begin{tabular}{|ccccccc|}
\hline \multirow{2}{*}{ Reaction } & \multicolumn{2}{c}{$v\left(\mathrm{~cm}^{-1}\right)$} & \multicolumn{2}{c}{$E_{a}\left(\mathrm{~kJ} \mathrm{~mol}^{-1}\right)$} & \multicolumn{2}{c}{$\Delta G^{\#}\left(\mathrm{~kJ} \mathrm{~mol}^{-1}\right)$} \\
& Gas & Water & Gas & Water & Gas & Water \\
$\mathrm{E} 1 \rightarrow \mathrm{K} 5$ & \multirow{2}{*}{-1813.03} & -1881.78 & 293.46 & 236.07 & 275.13 & 212.88 \\
$\mathrm{~K} 5 \rightarrow \mathrm{E} 1$ & & & 232.79 & 175.41 & 220.29 & 158.05 \\
$\mathrm{E} 2 \rightarrow \mathrm{K} 7$ & \multirow{2}{*}{-1791.89} & -1885.58 & 291.07 & 228.97 & 272.36 & 206.85 \\
$\mathrm{~K} 7 \rightarrow \mathrm{E} 2$ & & & 233.42 & 171.31 & 220.99 & 155.48 \\
$\mathrm{E} 2 \rightarrow \mathrm{K} 14$ & \multirow{2}{*}{-1819.46} & -1899.57 & 309.94 & 239.55 & 289.43 & 216.36 \\
$\mathrm{~K} 14 \rightarrow \mathrm{E} 2$ & & & 244.66 & 174.27 & 231.14 & 158.07 \\
$\mathrm{E} 3 \rightarrow \mathrm{K} 13$ & \multirow{2}{*}{-1805.39} & -1895.83 & 308.11 & 232.14 & 287.50 & 209.61 \\
$\mathrm{~K} 13 \rightarrow \mathrm{E} 3$ & & & 246.58 & 170.60 & 234.51 & 156.63 \\
\hline
\end{tabular}

The activation energies for the keto-enol tautomerism are up to 20 fold greater than those for the interconversions of the enediol-enediol or keto-keto forms, which may be explained by the fact that the mechanism of the keto-enol tautomerism is more complex and involves more atoms, as well as a geometrical rearrangement of the molecule. It should be mentioned here that $E_{a}$ and $\Delta G^{\#}$ are lower by $50-80 \mathrm{~kJ} \mathrm{~mol}^{-1}$ in water, than in gas, indicating that water influences the $\mathrm{H}$-transfer process, making it easier. The TSs structures reveal that the carboxylic oxygen that forms the hydrogen bond in the enediol structure is involved in the mechanism of proton transfer.

Table 5 summarizes kinetic and thermodynamic data for the transition states of isomerization and tautomerization reactions of the three most stable enediol forms, in the gas phase and in water. In the gas phase, E1 is the most stable species and isomerization reactions $\mathrm{E} 1 \leftrightarrow \mathrm{E} 2$ and E2 $\leftrightarrow \mathrm{E} 3$ are characterized by equilibrium constants of 0.13 . In water, the most stable isomer is E2, and isomerization reactions E1 $\leftrightarrow$ E2 and E2 $\leftrightarrow$ E3 are characterized by equilibrium constants of 1.21 and 0.61 , respectively. Results show that the presence of solvent (water) influences both, thermodinamic and kinetic parameters. In aqueous solution, the $\Delta E, \Delta \mathrm{H}, \Delta \mathrm{G}, \Delta G_{1}^{\#}$ and $\Delta G_{2}^{\#}$ decrease by around 2-7 kJ mol-1 in isomerization reactions and by around 10$30 \mathrm{~kJ} \mathrm{~mol}^{-1}$ in tautomerism reactions. In the gas phase, equilibrium constants of keto-enol transformations E1 $\leftrightarrow \mathrm{K} 5$, E2 $\leftrightarrow \mathrm{K} 7, \mathrm{E} 2 \leftrightarrow \mathrm{K} 14$ and E3 $\leftrightarrow \mathrm{K} 13$ equal to $2.46 \times 10^{-10}, 10.29 \times 10^{-10}, 0.61 \times 10^{-10}$ and $5.21 \times 10^{-10}$, respectively. In water, these values are of $1.41 \times 10^{-7}, 2.73 \times 10^{-7}, 5.61 \times 10^{-7}, 3.93 \times 10^{-7}$, respectively, showing a significant increase in the values of direct reactions. 
Table 5. Kinetic and thermodynamic data for the transition states of isomerization and tautomerization of the three most stable enediol forms, in the gas phase and in water. All energetic data have been reported in $\mathrm{kJ} \mathrm{mol}^{-1}$ and the rate constants in $\mathrm{s}^{-1}$. a

\begin{tabular}{|c|c|c|c|c|c|c|c|c|c|c|c|c|}
\hline & \multicolumn{4}{|c|}{ Isomerism } & \multicolumn{8}{|c|}{ Keto-enol tautomerism } \\
\hline & \multicolumn{2}{|c|}{$\mathrm{E} 1 \leftrightarrow \mathrm{E} 2$} & \multicolumn{2}{|c|}{$\mathrm{E} 2 \leftrightarrow \mathrm{E} 3$} & \multicolumn{2}{|c|}{$\mathrm{E} 1 \leftrightarrow \mathrm{K} 5$} & \multicolumn{2}{|c|}{$\mathrm{E} 2 \leftrightarrow \mathrm{K} 7$} & \multicolumn{2}{|c|}{$\mathrm{E} 2 \leftrightarrow \mathrm{K} 14$} & \multicolumn{2}{|c|}{$\mathrm{E} 3 \leftrightarrow \mathrm{K} 13$} \\
\hline & Gas & Water & Gas & Water & Gas & Water & Gas & Water & Gas & Water & Gas & Water \\
\hline$\Delta E$ & 5.18 & -0.59 & 5.24 & -0.01 & 60.67 & 35.61 & 57.66 & 39.97 & 65.28 & 35.84 & 61.54 & 39.78 \\
\hline$\Delta H$ & 5.26 & -0.53 & 5.27 & 0.58 & 59.55 & 40.44 & 56.50 & 42.57 & 63.70 & 35.38 & 59.77 & 42.12 \\
\hline$\Delta G$ & 5.07 & -0.48 & 4.98 & 1.24 & 54.84 & 39.07 & 51.30 & 37.42 & 58.29 & 35.64 & 52.99 & 36.52 \\
\hline Keq & 0.13 & 1.21 & 0.13 & 0.61 & $\begin{array}{c}2.46 x \\
10^{-10}\end{array}$ & $\begin{array}{c}1.41 \mathrm{x} \\
10^{-7}\end{array}$ & $\begin{array}{c}10.29 x \\
10^{-10}\end{array}$ & $\begin{array}{c}2.73 x \\
10^{-7}\end{array}$ & $\begin{array}{c}0.61 x \\
10^{-10}\end{array}$ & $\begin{array}{c}5.61 \mathrm{x} \\
10^{-7}\end{array}$ & $\begin{array}{c}5.21 x \\
10^{-10}\end{array}$ & $\begin{array}{c}3.93 x \\
10^{-7}\end{array}$ \\
\hline$\Delta G^{\#_{1}}$ & 47.50 & 39.90 & 47.01 & 40.19 & 275.13 & 261.20 & 272.36 & 260.72 & 289.43 & 270.23 & 287.50 & 267.22 \\
\hline$\Delta G^{\#}$ & 42.43 & 40.38 & 42.03 & 38.96 & 220.29 & 222.13 & 220.99 & 223.29 & 231.14 & 234.59 & 234.51 & 230.70 \\
\hline$k_{1}$ & $\begin{array}{c}2.96 \mathrm{x} \\
10^{4}\end{array}$ & $\begin{array}{c}6.29 x \\
10^{5}\end{array}$ & $\begin{array}{c}3.61 \\
\times 10^{4}\end{array}$ & $\begin{array}{r}55.98 \\
\times 10^{4}\end{array}$ & $\begin{array}{c}3.91 x \\
10^{-36}\end{array}$ & $\begin{array}{c}1.01 \mathrm{x} \\
10^{-33}\end{array}$ & $\begin{array}{c}1.19 x \\
10^{-35}\end{array}$ & $\begin{array}{c}1.23 x \\
10^{-33}\end{array}$ & $\begin{array}{c}1.22 \mathrm{x} \\
10^{-38}\end{array}$ & $\begin{array}{c}2.66 \mathrm{x} \\
10^{-35}\end{array}$ & $\begin{array}{c}2.66 \mathrm{x} \\
10^{-38}\end{array}$ & $\begin{array}{c}8.96 x \\
10^{-35}\end{array}$ \\
\hline$k_{2}$ & $\begin{array}{c}2.29 x \\
10^{5}\end{array}$ & $\begin{array}{c}5.18 x \\
10^{5}\end{array}$ & $\begin{array}{r}2.69 \\
\times 10^{5}\end{array}$ & $\begin{array}{r}9.19 \\
\times 10^{5}\end{array}$ & $\begin{array}{c}1.59 x \\
10^{-26}\end{array}$ & $\begin{array}{c}71.85 x \\
10^{-26}\end{array}$ & $\begin{array}{c}1.19 x \\
10^{-26}\end{array}$ & $\begin{array}{c}4.49 x \\
10^{-27}\end{array}$ & $\begin{array}{c}1.99 x \\
10^{-28}\end{array}$ & $\begin{array}{c}4.70 x \\
10^{-29}\end{array}$ & $\begin{array}{c}5.11 \mathrm{x} \\
10^{-29}\end{array}$ & $\begin{array}{c}2.26 \mathrm{x} \\
10^{-28}\end{array}$ \\
\hline
\end{tabular}

a $\Delta G^{\#}{ }_{1}=G_{T S}-G_{\text {reactant, }} \Delta G^{\#}{ }_{2}=G_{T S}-G_{\text {product, }} k_{1}$ - rate of forward reaction, $k_{2}$ - rate of reverse reaction.

\section{CONCLUSIONS}

In the course of this research, 45 isomers of dihydroxyfumaric acid have been identified, including 23 keto and 22 enediol forms, and their geometrical isomerization and tautomerization was studied at the B3LYP level of theory using the $6-311++G(2 d f, 2 p)$ basis set in the gas phase.

It was found that three enediol structures account for $99.96 \%$ of the dihydroxyfumaric acid in the gas phase, according to the following distribution: E1 - 87.4\%, E2 - 10.98\%, E3 - 1.4\%. In aqueous medium, these structures account for $97.3 \%$ of the acid, with the following relative abundance indices: E1 - 31.8\%, E2 - 38.4\%, E3 - 27.1\%.

The activation energy $\left(E_{a}\right)$, imaginary frequency $(v)$, and Gibbs free activation energy $\left(\Delta G^{\#}\right)$ were calculated for enediol-enediol and keto-keto interconversions. These interconversions proceed through internal rotation $\left(E_{a} \approx 0.15-75 \mathrm{~kJ} \mathrm{~mol}^{-1}\right)$, because proton transfer requires a significantly higher activation energy $\left(E_{a} \approx 135-160 \mathrm{~kJ} \mathrm{~mol}^{-1}\right)$, in the gas phase and aqueous solution. 
Keto-enol tautomeric reactions of dihydroxyfumaric acid were investigated for the three most stable enediol structures. The activation energies of the keto-enol interconversions are in the range of $230-310 \mathrm{~kJ}$ $\mathrm{mol}^{-1}$ in the gas phase, which is on average, up to 20-folds greater than the activation energies of the enediol or keto interconversions between themselves. In water, this process unfolds easier, and $E_{a}$ and $\Delta G^{\#}$ are lower by $50-80 \mathrm{~kJ}$ $\mathrm{mol}^{-1}$. Kinetic and thermodynamic calculations were performed for the transition states of isomerization and tautomerization reactions of the three most stable enediol forms, in the gas phase and in water, equilibrium constants have been identified, along with the forward and reverse reaction rates.

In conclusion, this research presents important information regarding the isomerization and tautomeriztion reactions of difydroxyfumaric acid, which broaden the understanding of these processes in gas and water.

\section{THEORETICAL APPROACH AND METHODS}

All geometry optimizations were done by density functional theory (DFT), which give reliable thermodynamic data for molecules and systems with hydrogen bonding, when employed with a large and reasonable basis set [14]. Therefore, the geometries of all possible tautomers-rotamers of DHF and the relevant transition states (TSs) were optimized at the B3LYP/6$311++G(2 d f, 2 p)$ level. All calculations were carried out using the ORCA quantum chemistry package [15], without any symmetry constraints. The absence of imaginary frequencies in the vibrational spectra confirmed that calculated isomer structures were energy minimums.

The Nudged Elastic Band method (as implemented in the ORCA software [15]) was used as a first step to find TSs, which were further optimized and studied at the B3LYP/6-311++G(2df,2p) level, as mentioned above. Transition states were verified with frequency calculations, and were characterized by the existence of only one imaginary frequency for motion along the reaction coordinate.

The effect of water, as the solvent, on the isomerism and tautomerism reactions was calculated using the SMD model, as implemented in ORCA [15].

The Boltzmann equation (Eq.1) was used to calculate the relative abundances (RA) of the enediol and keto isomers, using the internal energy corrected for the zeropoint energy (ZPE).

$$
R A=\exp (-\Delta E / R T)
$$

where $\Delta E=\Delta E_{e l}+\Delta Z P E$. 

equation

Rate constants were calculated by canonical TS theory using Eyring

$$
k=\frac{k_{B} T}{h} e^{-\Delta G^{\#} / R T}
$$

where $\Delta G^{\#}$ is the Gibbs energy of activation, $k_{B}$ is Boltzmann's constant, and $h$ is Planck's constant.

The equilibrium constant $K_{e q}$ is given by the following equation:

$$
K_{e q}=\exp (-\Delta G / R T)
$$

where $\Delta G=G_{298}^{\text {products }}-G_{298}^{\text {reactants }}$ and individual thermodynamic parameters are $G_{298}=H_{298}-T S_{298}, H_{298}=E_{e l}+E_{v i b}+E_{\text {rot }}+E_{\text {trans }}+Z P E+k_{B} T$ and $S_{298}=S_{e l}+S_{\text {vib }}+S_{\text {rot }}+S_{\text {trans }}$. All calculations were carried out at $298.15 \mathrm{~K}$ and $1.0 \mathrm{~atm}$.

\section{AUTHOR CONTRIBUTIONS}

Natalia Bolocan: Conceptualization, investigation, writing-original draft preparation and editing.

Gheorghe Duca: Conceptualization, supervision, writing - review and editing.

\section{FUNDING INFORMATION}

National Research Project REDOX No. 20.80009.5007.04

\section{REFERENCES}

1. a) H.J.H. Fenton; J. Chem. Soc. Trans., 1894, 65, 899 - 910; b) H. J. H. Fenton, J. Chem. Soc. Trans., 1905, 87, $804-818$.

2. A. Eschenmoser; Chem. Biodivers., 2007, 4, $554-573$.

3. A. Sychev; G. Duca; Fruit growing, Viticult. vinif. Mold., 1985, 12, 39-41 (in Romanian).

4. G. Duca; Homogeneous Catalysis with Metal Complexes: Fundamentals and Applications; Springer-Verlag Berlin Heidelberg, 2012; Vol. XII, 478 p.

5. I. Stepanov; S.G. Carmella; S.S. Hecht; G. Duca; J. Agric. Food Chem., 2002, 50 (10), 2793-2797.

6. Gh. Duca; Free radicals in natural water, in Free Radicals in Biology and Environment; F. Minisci Ed.; Springer Dordrecht, NATO ASI Series, 1997, pp. 475-489.

7. D. Porubin; Chem. J. Mold., 2007, 2, 3-7. 
8. D. Porubin; S. Hecht; ZZ. Li; M. Gonta; I. Stepanov; J. Agric. Food. Chem., 2007, 55 (17), 7199-7204.

9. N. Secara; G. Duca; L. Vlad; F. Macaev; Chem. J. Mold., 2010, 5 (2), 59-67.

10. E.D. Raczynska; W. Kosinska; B. Osmiałowski; R. Gawinecki; Chem. Rev. 2005, 105, 3561-3612.

11. P. Souchay; D. Fleury; M. Fleury; C. R. Acad. Sc. Paris, 1967, 264, C, $2130-$ 2133.

12. D. Sazou; P. Karabinas; D. Jannakoudakis; J. Electroanal. Chem., 1984, 176, 225-234.

13. S.O. Travin; T.D. Kemp; P. Mur; Khim. Fiz. (USSR), 1986, 5 (10), 1393-1404.

14. J. Tirado-Rives; W. Jorgensen; J. Chem. Theory Comput., 2008, 4, 297-306.

15. F. Neese; F. Wennmohs; U. Becker; C. Riplinger; J. Chem. Phys., 2020, 152, 224108.

16. M.P. Andersson and P. Uvdal, J. Phys. Chem. A, 2005, 109 (12), 2937-2941. 
